\title{
Could certain mouthwashes reduce transmissibility of COVID-19?
}

\section{Fatima Elmahgoub*1 and Yasmine Coll ${ }^{2}$}

\section{A commentary on}

\section{Cavalcante-Leão B L, de Araujo C-M, Basso I-B et al.}

Is there scientific evidence of the mouthwashes effectiveness in reducing viral load in Covid-19? A systematic review. J Clin Exp Dent 2021; DOI: $10.4317 /$ jced.57406.

\begin{abstract}
Data sources Six electronic databases were searched including PubMed/Medline, Embase, Web of Science, Scopus, Cochrane, and Latin American and Caribbean Literature in Health Science (LILACS). Grey literature was also examined. There were no restrictions with regards to language, time of publication or participant demographics including gender, age and ethnicity.

Study selection Both clinical and in vitro studies were included in the data search. Inclusion and exclusion criteria were clearly identified. Study selection was carried out in two phases by two independent reviewers. The studies included fitted the following criteria: studies that hypothesised the use of mouthwashes as a form of intervention to decrease the viral load in saliva contaminated with coronavirus. Two other studies were included in this review and both were conducted in Germany, performed in vitro, which tested povidone-iodine (PVP-I) mouthwash at two different concentrations: $1 \%$ and $7 \%$. Both studies used the same culture mediums for clean and dirty conditions, and the evaluated outcome was the viral titre of MERS-CoV and SARS-CoV. The studies were published in 2015 and 2018. Neither study evaluated the action of this mouthwash on SARS-CoV-2 infection.
\end{abstract}

Data extraction and synthesis The extraction of data followed the Preferred Reporting Items for Systematic Reviews and Meta-Analyses (PRISMA) guideline process. Studies chosen for analysis included assessment of the following data parameters for risk of bias: cell origin and cell type used, duration of exposure, frequency of exposure, magnetic flux density of exposure, environmental background magnetic field, use of control treatment, temperature control, blinding of exposure, randomisation of exposure, measurement of cell vitality, identical methods for control and exposure groups, randomisation of data measurements and potential industry sponsorship.

Results One study assessed the effect of the mouthwash on MERS$\mathrm{COV}$ and the other on both MERS-CoV and SARS-CoV. In both studies, following 15 seconds of exposure to the mouthwash, a reduction in viral load of $>99.99 \%$ was seen. There was a measurable reduction in viral titre in the samples, with one study reporting a reduction to $4.30 \log 10 \mathrm{TCID} 50 / \mathrm{ml}$ from $6.00-6.50 \log 10 \mathrm{TCID} 50 / \mathrm{ml}$. Other mouthwashes such as hydrogen peroxide and chlorhexidine were not assessed in these studies.

Conclusions The study concludes that PVP-I mouthwash at concentrations of $1 \%$ and $7 \%$ for 15 seconds may be effective at

\section{GRADE rating}

Practice point

- This paper explores an interesting area regarding the possibility of reducing transmissibility of COVID-19 using PVP-I mouthwash; however, there is insufficient evidence to draw conclusions from this study and further research is required.

reducing the viral load of COVID-19 in human saliva. The level of scientific evidence, however, is low.

\section{Commentary}

The current ongoing coronavirus disease (COVID-19) pandemic is caused by the SARS-CoV-2 virus. The virus is transmitted via droplets through contact of mucous membranes with contaminated surfaces, as well as via aerosols and aerosol generating procedures. ${ }^{1}$ Saliva is an important reservoir of SARS-CoV-2, and studies suggest that the viral load in infected individuals can be up to 107 per $\mathrm{ml}^{2}{ }^{2}$

Various studies have attempted to assess the potential effects of several mouthwashes on COVID-19 transmissibility. ${ }^{2,3,4}$ This review aimed to identify whether there was evidence regarding a reduction in the viral load found in saliva following the use of three types of mouthwashes: chlorhexidine, hydrogen peroxide and povidone-iodine (PVP-I). Such evidence may be useful both in a clinical dental setting as well as confined public spaces, to reduce the risk of person-to-person transmissibility.

The literature search was found to be acceptable as six databases were explored, as well as grey literature, and there was no restriction on the language of studies included. In this review, the authors have followed the PRISMA guidelines and have registered the protocol with PROSPERO. The database search strategies were included within the article and the population, intervention, comparison and outcomes were clearly identified. A manual search of the literature was carried out to detect articles which addressed forms of prevention in dentistry in relation to COVID-19 and expert opinion was also sought in this search for relevant studies. Studies with incomplete data were excluded.

This review was predominantly based on in vitro studies. Study selection was carried out in two phases: in the first phase, titles and abstracts were reviewed and 1,222 articles were identified and retrieved; duplicate articles were then removed. In the second phase, studies that met the eligibility criteria were chosen for full-text analysis. In total, ten articles were selected for complete reading. The reviewers were shielded with a third member acting as a moderator, to reduce bias. Where there was disagreement, a third reviewer was involved in decision-making and Cohen's kappa was used to measure inter-reviewer agreement. The initial search included clinical trials and in vitro studies; however, only two in vitro experimental studies fit the required criteria, with both 
identified studies focusing on PVP-I mouthwash and its effects on the viral load of MERS-CoV and SARS-CoV. Neither one of these studies assessed the efficacy of chlorhexidine or hydrogen peroxide mouthwashes in reducing the salivary viral load and neither study assessed the effectiveness of any mouthwash specifically on SARS-CoV-2, as they were conducted in 2015 and 2018, before the emergence of COVID-19.

In vitro studies are carried out in controlled environments, which reduces the risk of bias. The authors attempted to assess the quality of the studies by using an adaptation based on a previous study, as there is no standard tool for assessment of risk of bias for in vitro studies. The studies were assessed for reporting quality, performance bias, selection bias, detection bias and other sources of bias, with a focus on the assessment of 13 parameters for risk of bias. None of the items assessed received a 'high risk of bias'. The studies were categorised as having 'low risk of bias' for 8 out of 13 items, with the remaining items displaying 'unclear bias', particularly higher in relation to the reporting quality and performance bias categories.

The results of the included studies were not clearly displayed and therefore could not further be analysed. The overall results display a reduction of viral load of $>99.99 \%$; however, there were no details of how these results were derived. The sample size was not mentioned and information on sample preparation and handling, allocation sequence, randomisation and blinding was not clearly stated.

The possible impact of PVP-I and other mouthwashes in reducing the viral load within the oral cavity could potentially affect the severity of symptoms of disease as well as transmissibility, which makes this an entirely relevant and interesting area to explore. More attention has been placed on the use of personal protective equipment such as masks to combat the transmission of COVID-19, with less attention being placed on methods of controlling viral load. Specifically, within dental environments, aerosol generating procedures lead to a higher risk of transmission and theoretically dentists may be able to reduce these risks.

According to the pyramid of evidence, in vitro studies produce the lowest reliable evidence, and although these types of studies form a large proportion of dental research, frequently to assess the properties of dental materials, the importance of these lie in how they can translate onto a clinical setting. The authors have attempted to identify studies with clear and specific eligibility criteria; however, there are limitations to the study design which the authors have addressed, including the lack of data regarding different common mouthwashes, the lack of data specific to assessing the effectiveness on SARS-CoV-2 and the requirement of more research into this area, specifically randomised clinical trials. As such, until further research is carried out in this area, one cannot conclude that PVP-I or other mouthwashes can satisfactorily reduce the risk of transmission of COVID-19.

\section{Author affiliations}

${ }^{1}$ DCT3 OMFS, University Hospital Crosshouse, Kilmarnock, UK; ${ }^{2}$ DCT2 OMFS, University Hospital Crosshouse, Kilmarnock, UK. ${ }^{*}$ Correspondence to: Fatima Elmahgoub

\section{References}

1. Jayaweera M, Perera H, Gunawardana B, Manatunge J. Transmission of COVID19 virus by droplets and aerosols: A critical review on the unresolved dichotomy. Environ Res 2020; 188: 109819.

2. Kalsi H, Edwards D. PVP-I mouth rinse in dental practice. Br Dent / 2020; 229: 736.

3. Vergara-Buenaventura A, Castro-Ruiz C. Use of mouthwashes against COVOD-19 in dentistry. Br / Oral Maxillofac Surg 2020; 58: 924-927.

4. Carrouel F, Gonçalves L S, Conte M P et al. Antiviral Activity of Reagents in Mouth Rinses against SARS-CoV-2. J Dent Res 2021; 100: 124-132.

Evidence-Based Dentistry (2021) 22, 82-83.

https://doi.org/ 10.1038/s41432-021-0172-4 